\section{Case Reports in Neurology}

\title{
Limb-Shaking Transient Ischemic Attacks in a Patient with Previous Bilateral Neck Irradiation: The Role of Collateral Flow
}

\author{
Chai-Hoon Nowel Tan ${ }^{a} \quad$ Manish Taneja ${ }^{b}$ \\ Narayanaswamy Venketasubramanian ${ }^{\mathrm{b}}$ \\ aYong Loo Lin School of Medicine, National University of Singapore, \\ Singapore, Singapore; ${ }^{b}$ Raffles Neuroscience Centre, Raffles Hospital \\ Singapore, Singapore
}

\section{Keywords}

Limb-shaking transient ischemic attack - Carotid artery occlusion · Neck radiation . Nasopharyngeal carcinoma $\cdot$ Stroke

\begin{abstract}
Nasopharyngeal carcinoma (NPC) is commonly treated with bilateral neck radiation, which is closely associated with the complication of carotid-occlusive disease. This leads to cerebral hemodynamic compromise and possible ischemic stroke. Another manifestation is limb-shaking transient ischemic attacks (LS-TIAs), characterized by rhythmic jerks which can be easily mistaken as a focal motor seizure. We describe a case of unilateral LS-TIAs from bilateral carotid occlusion that resolved with contralateral carotid revascularization. Our patient is a 65year-old gentleman who had no significant co-morbidities other than a past history of bilateral neck irradiation for NPC 8 years before. He presented with left-sided limb weakness and subsequently left-sided limb involuntary movements whenever he sat up or stood. His symptoms did not respond to anti-epileptic therapy. Clinical and neurological examination was significant for a left pronator drift and weak left finger abduction. Computed tomography and magnetic resonance imaging of the brain revealed infarcts in the right periventricular and watershed areas; MR angiogram showed bilateral internal carotid artery occlusion. Single photon
\end{abstract}

Narayanaswamy Venketasubramanian
Raffles Neuroscience Centre, Raffles Hospital
585 North Bridge Road, \#09-00 Raffles Specialist Centre
Singapore 188770 (Singapore)
drnvramani@gmail.com




\section{Case Reports in Neurology}

Case Rep Neurol 2020;12:84-90

\begin{tabular}{l|l}
\hline DOI: 10.1159/000505391 & @ 2020 The Author(s). Published by S. Karger AG, Basel
\end{tabular} www.karger.com/crn

Tan et al.: Limb-Shaking TIAs after Neck Irradiation

emission computed tomography showed reduced blood flow in the right frontal, temporal, and parietal regions, that reduced further after acetazolamide challenge. He was diagnosed as having LS-TIA secondary to carotid-occlusive disease. Attempts at endovascular opening the right internal carotid artery failed. Following successful left carotid angioplasty and stenting, his symptoms gradually resolved. The left internal carotid artery remained patent at the 3month follow-up; the right side remained occluded. Our case supports the hypothesis that LSTIAs are due to hemodynamic compromise and may respond to improved collateral cerebral blood flow.

(C) 2020 The Author(s)

Published by S. Karger AG, Basel

\section{Introduction}

Brain ischemia resulting in transient ischemic attacks (TIAs) or stroke typically present with neurological deficits such as hemiparesis, hemisensory loss, visual field defects, or aphasia. On the other hand, seizures usually present with involuntary movements secondary to neural hyperexcitability. Limb-shaking TIA (LS-TIA) presents with focal involuntary jerks that are not typical features of focal cerebral infarction or seizure. It is important to diagnose this entity as it is an indicator of severe carotid-occlusive disease [1]. The pathology may involve either the intracranial or extracranial carotid arteries. Appropriate treatment, such as extracranial-intracranial (EC-IC) bypass, may reduce the risk of stroke and minimize the recurrence of this hyperkinetic phenomenon [2]. We report a case of a patient with previous nasopharyngeal carcinoma (NPC) who had received neck irradiation, who subsequently presented with minor stroke and LS-TIAs that responded to contralateral revascularization.

\section{Case Report}

Our patient, a 65-year-old Chinese gentleman, presented initially to his family doctor with left-sided weakness and was diagnosed with stroke. Over time, his power improved and was close to achieving full recovery when he developed involuntary left limb jerks and drowsiness, especially when he sat up. He had no co-morbidities such as hypertension, diabetes, hyperlipidemia, coronary artery disease, or peripheral vascular disease. He had smoked for 1-2 years during his youth. He had been diagnosed with NPC 8 years earlier after presenting with hoarseness of voice and had received bilateral neck irradiation. Clinically, he was well. He had no dysphagia, anopia, or neglect, and no ocular or cervical bruits. He had a mild left lower facial weakness, left pronator drift with mild hyperreflexia, but full power, coordination, and sensation.

CT and MRI of the brain revealed infarcts in the right periventricular and watershed areas (Fig. 1). MR angiography showed bilateral internal carotid artery occlusion (Fig. 2). Single photon emission computed tomography (SPECT) showed reduced blood flow in the right frontal, temporal, and parietal regions, that further reduced after acetazolamide challenge (Fig. 3). These imaging features were suggestive of cerebral hypoperfusion and a diagnosis of "limb-shaking transient ischemic attacks" was made. His previous history of bilateral neck radiation was suspected to be the cause of his bilateral carotid-occlusive disease. EEG was normal.

\section{Karger'=}




\section{Case Reports in Neurology}

Case Rep Neurol 2020;12:84-90

\begin{tabular}{l|l}
\hline DOI: 10.1159/000505391 & (c) 2020 The Author(s). Published by S. Karger AG, Basel \\
\hline
\end{tabular} www.karger.com/crn

Tan et al.: Limb-Shaking TIAs after Neck Irradiation

Attempts were made on the right internal carotid artery, but they were unsuccessful. Subsequently, the patient successfully underwent left internal carotid angioplasty and stenting. Post-procedure, his left-sided jerking movements were reduced, and he no longer suffered reduction in consciousness. At 3 months post-procedure, follow-up carotid angiography showed patent left carotid artery and persistent right-sided carotid occlusion, with no further TIAs (Fig. 4).

\section{Discussion/Conclusion}

Irradiation-induced extracranial carotid-occlusive disease is a well-known complication of NPC treatment with radiotherapy [3], which remains as the standard treatment for nonmetastatic disease. A retrospective study demonstrated a significantly higher proportion of carotid artery disease in NPC patients compared to unselected first-ever Taiwanese stroke patients, with carotid artery disease and absence of diabetes mellitus as the strongest independent discriminators between these two groups of patients. This finding strengthens the hypothesis that irradiation-induced endothelial damage initiates the process of accelerated atherosclerosis independently from other vascular risk factors like diabetes mellitus [4]. Possible mechanisms include insult to intima media which worsens atherosclerosis, and adventitia that injures the vasa vasorum. Our patient did not have a history of vascular risk factors other than a remote history of smoking briefly in his youth. He only had neck irradiation for NPC 8 years before presentation.

LS-TIA, which was first described by Fisher [5] in 1962 are transient attacks of repetitive, involuntary hyperkinetic limb movements. While this hyperkinetic phenomenon is a source of diagnostic confusion with seizures, clues such as absence of Jacksonian march, absence of incontinence, as well as normal EEG help to differentiate the two and point the diagnosis towards LS-TIA [6]. The symptom is described to be precipitated by standing up, hyperextending the neck, improvement on sitting or lying down, and associated with dizziness [7]. Although the exact mechanism and site of involvement of this phenomenon is unclear, the above clinical presentation supports the "hemodynamic compromise" theory. Carotid-occlusive disease or high-grade carotid stenosis results in decreased cerebral blood flow and cerebral hypoperfusion in certain critical territories. In a case-control study, patients with limb-shaking more frequently had symptoms precipitated by exercising, recurrent ischemic deficits after documented internal carotid artery occlusion, more often had leptomeningeal collaterals on contrast angiograms, and lower carbon-dioxide reactivity measured by transcranial Doppler [8].

A near-infrared spectroscopy study (fNIRS) that allows for noninvasive monitoring of bedside cortical changes in oxyhemoglobin $\left(\mathrm{HbO}_{2}\right)$, deoxyhemoglobin $(\mathrm{HbR})$, and total hemoglobin ( $\mathrm{HbT}$, as a proxy to cerebral blood volume) reported decreased cerebral blood flow to the middle cerebral artery territory as the patient presented with orthostatic hypotension and limb-shaking phenomenon [9].

In addition, molecular imaging modalities like SPECT and PET allow for evaluation of cerebral blood flow with the use of radioactive tracers. Oxygen extraction fraction of cerebral hemispheres, which is measured with PET scan, is used for prognostication and to direct management of carotid-occlusive disease [10]. An increased oxygen extraction fraction is suggestive of misery perfusion syndrome, representing a critical stage where cerebral blood supply is insufficient to meet metabolic demand. Studies have shown that misery perfusion remains

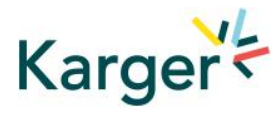




\section{Case Reports in Neurology}

Tan et al.: Limb-Shaking TIAs after Neck Irradiation

a predictor of subsequent stroke in carotid-occlusive disease [11]. Alternatively, cerebrovascular reactivity (CVR) in response to acetazolamide can be detected by SPECT to assess for risk of misery perfusion in clinical practice. Studies have shown that reduction in CVR to acetazolamide, as measured by SPECT, is also significantly associated with increased risk of stroke recurrence in patients with major carotid-occlusive disease [12]. However, it is still controversial as to whether impaired CVR and oxygen extraction fraction are linearly and inversely correlated based on differing results across different studies [13]. This has important implications on the sensitivity in the detection of patients who are at higher stroke risk secondary to carotid-occlusive disease.

The management of this atypical presentation of TIA hence aims to re-establish or improve cerebral blood flow and can be divided into medical and surgical methods. Careful control of co-morbidities - modification of anti-hypertensive control in particular - may be considered in patients who decline surgery or are poor surgical candidates. Surgical revascularization options include endarterectomy, angioplasty with stenting, and external carotid-internal carotid (EC-IC) bypass depending on patient and disease factors. Successful carotid endarterectomy [14] has been documented in multiple case reports for the treatment of LSTIA. However, carotid endarterectomy is confounded by the need to operate across scarred tissue planes. As a result, patients who are at high surgical risk may consider angioplasty and stenting. As for EC-IC bypass, a recent randomized clinical trial did not reduce the incidence of subsequent ischemic stroke [15]. However, there are currently no such trials for patients with LS-TIA specifically.

In our patient, we were unable to directly revascularize his symptomatic hemisphere despite several attempts. However, his contralateral occluded carotid artery was re-opened successfully. Over time, his symptoms resolved. It is likely that reopening the contralateral artery allowed improved collateral flow to the symptomatic hemisphere, and thus his symptoms resolved.

In conclusion, LS-TIAs are usually associated with carotid-occlusive disease, which is a common complication of NPC treatment with radiotherapy. Cerebral revascularization can reduce symptoms. In our case, the patient described here was successfully treated with contralateral carotid angioplasty and stenting with positive outcome of vessel patency and reduction in symptoms, supporting the value of enhancing collateral flow if direct revascularization is not possible.

\section{Statement of Ethics}

This research complies with the guidelines for human studies and was conducted ethically in accordance with the World Medical Association Declaration of Helsinki. The patient provided written informed consent to publish the case (including publication of images).

\section{Conflict of Interest Statement}

The authors have no conflicts of interest to declare.

\section{Karger' ${ }^{\prime \prime}$}




\section{Case Reports in Neurology}

\section{Funding Sources}

There are no funding sources to disclose.

\section{Author Contributions}

Chai-Hoon Nowel Tan: prepared the manuscript.

Manish Taneja: conceived the project, as the radiologist performed the cerebral angiogram and stenting, and reviewed the manuscript.

Narayanaswamy Venketasubramanian: conceived the project, was the principal clinician for the patient, guided the first author, and reviewed the manuscript.

\section{References}

1 Yoon Y, Kim JS. Limb-shaking TIA: an asterixis. Neurology. 2013 Sep;81(10):931-2.

2 van Loon J, Wilms G, Thijs V. STA-MCA Revisited: Treatment of Limb-Shaking TIA. World Neurosurg. 2012 Jan;77(1):211-2.

3 Lam WW, Leung SF, So NM, Wong KS, Liu KH, Ku PK, et al. Incidence of carotid stenosis in nasopharyngeal carcinoma patients after radiotherapy. Cancer. 2001 Nov;92(9):2357-63.

4 Li CS, Schminke U, Tan TY. Extracranial carotid artery disease in nasopharyngeal carcinoma patients with post-irradiation ischemic stroke. Clin Neurol Neurosurg. 2010 Oct;112(8):682-6.

5 Fisher CM. Concerning recurrent transient cerebral ischemic attacks. Can Med Assoc J. 1962 Jun;86:1091-9.

6 Baquis GD, Pessin MS, Scott RM. Limb shaking - a carotid TIA. Stroke. 1985 May-Jun;16(3):444-8.

7 Ali S, Khan MA, Khealani B. Limb-shaking Transient Ischemic Attacks: case report and review of literature. BMC Neurol. 2006 Jan;6(1):5.

8 Persoon S, Kappelle LJ, Klijn CJ. Limb-shaking transient ischaemic attacks in patients with internal carotid artery occlusion: a case-control study. Brain. 2010 Mar;133(Pt 3):915-22.

9 Kassab A, Tremblay J, Poppe AY, Létourneau-Guillon L, Gallagher A, Nguyen DK. Cerebral hemodynamic changes during limb-shaking TIA: A near-infrared spectroscopy study. Neurology. 2016 Mar;86(12):1166-8

10 Yamauchi H, Fukuyama H, Nagahama Y, Nabatame H, Ueno M, Nishizawa S, et al. Significance of increased oxygen extraction fraction in five-year prognosis of major cerebral arterial occlusive diseases. J Nucl Med. 1999 Dec;40(12):1992-8.

11 Yamauchi H, Higashi T, Kagawa S, Nishii R, Kudo T, Sugimoto K, et al. Is misery perfusion still a predictor of stroke in symptomatic major cerebral artery disease? Brain. 2012 Aug;135(Pt 8):2515-26.

12 Ogasawara K, Ogawa A, Yoshimoto T. Cerebrovascular reactivity to acetazolamide and outcome in patients with symptomatic internal carotid or middle cerebral artery occlusion: a xenon-133 single-photon emission computed tomography study. Stroke. 2002 Jul;33(7):1857-62.

13 Kuroda S, Shiga T, Houkin K, Ishikawa T, Katoh C, Tamaki N, et al. Cerebral oxygen metabolism and neuronal integrity in patients with impaired vasoreactivity attributable to occlusive carotid artery disease. Stroke. 2006 Feb;37(2):393-8.

14 Siniscalchi A, Gallelli L, Malferrari G, De Sarro G. Limb-shaking transient ischemic attack associated with focal electroencephalography slowing: case report. J Vasc Interv Neurol. 2012 Jun;5(1):3-5.

15 Powers WJ, Clarke WR, Grubb RL Jr, Videen TO, Adams HP Jr, Derdeyn CP, et al.; COSS Investigators. Extracranial-intracranial bypass surgery for stroke prevention in hemodynamic cerebral ischemia: the Carotid Occlusion Surgery Study randomized trial. JAMA. 2011 Nov;306(18):1983-92. 
Case Reports in Neurology
Case Rep Neurol 2020;12:84-90 DOI: 10.1159/000505391 (c) 2020 The Author(s). Published by S. Karger AG, Basel Tan et al: Limb-Shaking TIAs after Neck Irradiation

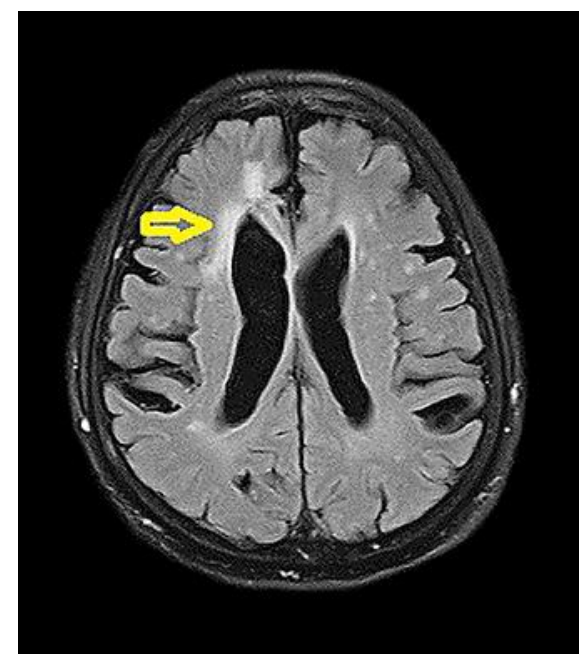

Fig. 1. Brain MRI (FLAIR) showing infarct in the right periventricular region.

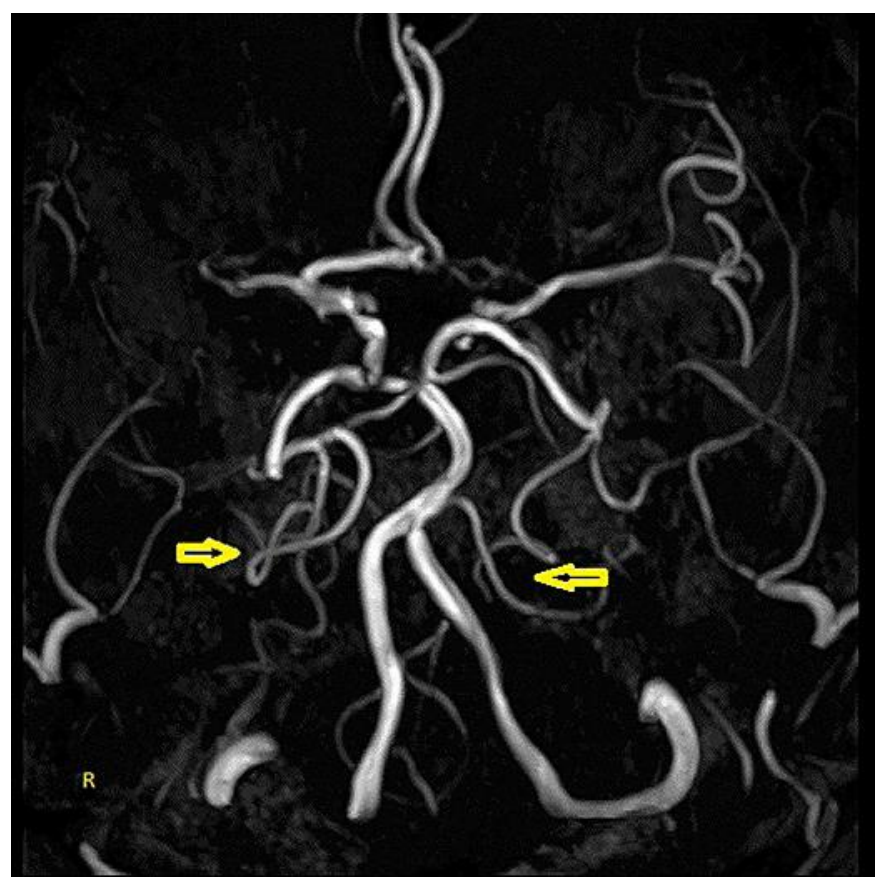

Fig. 2. MR angiography showing bilateral internal carotid artery occlusion. 
Case Reports in Neurology
Case Rep Neurol 2020;12:84-90

DOI: 10.1159/000505391

(C)

2020 The Author(s). Published by S. Karger AG, Basel

Tan et al.: Limb-Shaking TIAs after Neck Irradiation

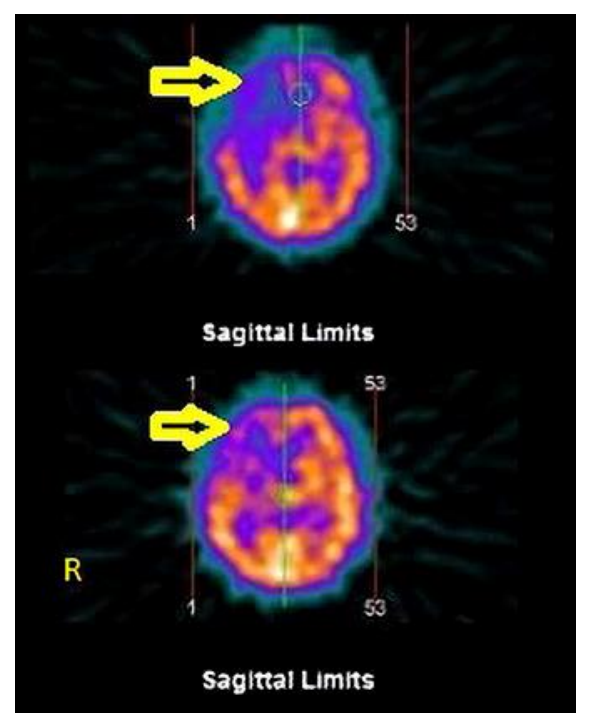

Fig. 3. Single photon emission computed tomography showing further reduction in blood flow to the right frontal parietal and temporal regions after acetazolamide challenge (top panel), compared to baseline (bottom panel).

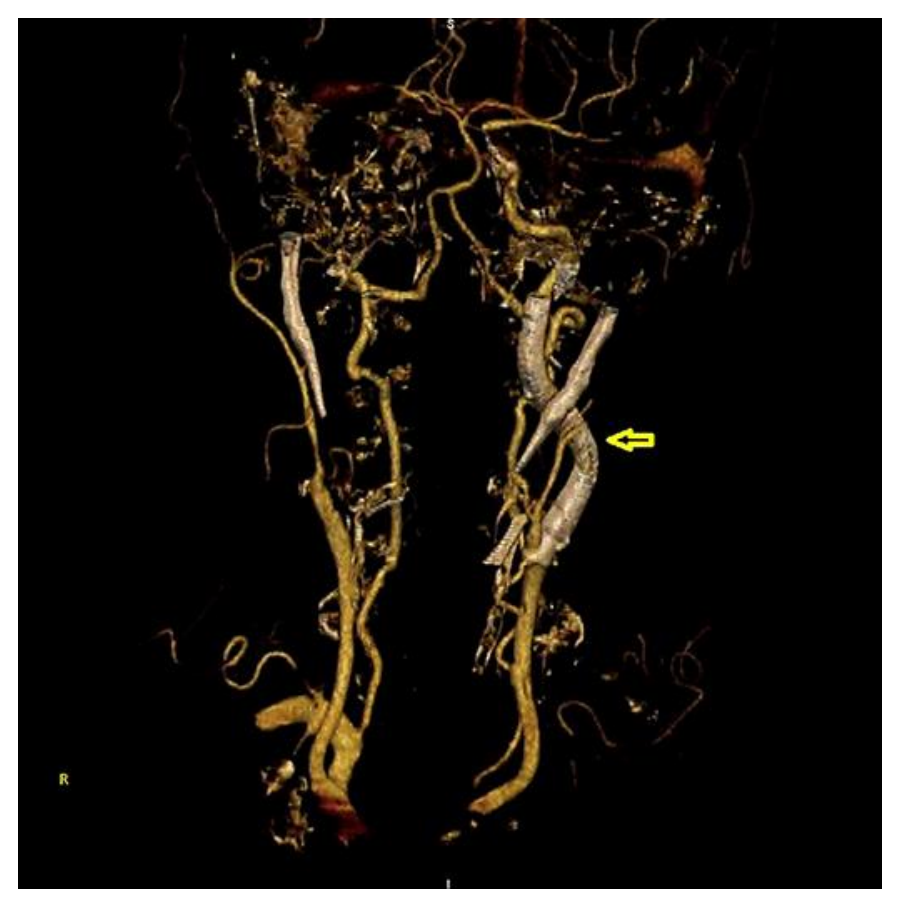

Fig. 4. MR angiography showing patent left internal carotid artery at 3 months post-stenting.

\section{Karger'}

